\title{
Stock Market Prediction Using Machine Learning
}

\author{
Yogita Deshmukh ${ }^{1}$, Deepmala Saratkar ${ }^{2}$, Harshal Hiratkar ${ }^{3}$, Sudhanshu Dhopte ${ }^{4}$, Swapnil Patankar ${ }^{5}$, \\ Triveni Jambhulkar ${ }^{6}$, Yash Tiwari ${ }^{7}$ \\ Assistant Professor, Computer Technology, RGCER, Nagpur, Maharashtra, India ${ }^{1}$ \\ U.G. Student, Computer Technology, RGCER, Nagpur, Maharashtra, India ${ }^{2,3,4,5,6,7}$
}

\begin{abstract}
The stock market has always been a promising avenue for lucrative investing, but most of the profit making depends on the analysis of the current and past market scenario followed by subsequent predictive actions. The currently overblown market economy has given rise to numerous variables which need to be considered before making a beneficial transaction in the stock market. Manually analysing all these variables and affecting factors is too cumbersome and error prone. Therefore, a Machine Learning approach is best suited for analysis of such a seemingly chaotic system. In this project we are using Machine learning, which give a prediction of various aspects of a particular stock or an index, such as future values of the opening price, closing price, index value etc. This will help investors and traders make better and faster decisions.
\end{abstract}

Keywords: Stock market; Prediction; Machine learning; Artificial neural network

\section{INTRODUCTION}

Stock Market prediction and analysis is the act of trying to determine the future value of a company stock or other financial instrument traded on an exchange. Stock market is the important part of economy of the country and plays a vital role in the growth of the industry and commerce of the country that eventually affects the economy of the country. Both investors and industry are involved in stock market and wants to know whether some stock will rise or fall over certain period of time. The stock market is the primary source for any company to raise funds for business expansions. It is based on the concept of demand and supply. If the demand for a company's stock is higher, then the company share price increases and if the demand for company's stock is low then the company share price decrease. Due to involvement of many number of industries and companies, it contain very large sets of data from which it is difficult to extract information and analyse their trend of work manually. Stock market analysis and prediction will reveal the market patterns and predict the time to purchase stock. The successful prediction of a stock's future price could yield significant profit. This is done using large historic market data to represent varying conditions and confirming that the time series patterns have statistically significant predictive power for high probability of profitable trades and high profitable returns for the competitive business investment. Machine learning is a method of data analysis that automates analytical model building. Using algorithms that iteratively learn from data, machine learning allows computers to find hidden insights without being explicitly programmed where to look. Application of Machine learning algorithms is generally focused on technical analysis but incorporation of the concepts of fundamental analysis into machine learning can be beneficial. This project tells about how various efforts have been taken in the application of Machine Learning to Stock forecasting and also suggests new potent ideas that can be worked upon. Recently, a lot of interesting work has been done in the area of applying Machine Learning Algorithms for analysing price patterns and predicting stock prices and index changes. Most stock traders nowadays depend on Intelligent Trading Systems which help them in predicting prices based on various situations and conditions, thereby helping them in making instantaneous investment decisions.

\section{LITERATURE SURVEY}

In the last few decades forecasting of stock returns has become an important field of research. In most of the cases the researchers had attempted to establish a linear relationship between the input macroeconomic variables and the stock returns. After the discovery of nonlinearity in the stock market index returns, many literatures have come up in nonlinear statistical modelling of the stock returns, most of them required that the nonlinear model be specified before the estimation is done. But since stock market return is noisy, uncertain, chaotic and nonlinear in nature, ANN has evolved out to be better technique in capturing the structural relationship between a stock's performance and its determinant factors more accurately than many other statistical techniques. In literature, different sets of input variables are used to predict stock returns. In fact, different input variables are used to predict the same set of stock return data. 
Vol. 8, Issue 1, January 2019

Some researchers used input data from a single time series where others considered the inclusion of heterogeneous market information and macro-economic variables. Some researchers even pre-processed these input data sets before feeding it to the ANN for forecasting.

\section{RELATED WORK}

Min and Lee [1] were doing prediction of bankruptcy using machine learning. They evaluated methods based on Support Vector Machine, multiple discriminant analysis, logistic regression analysis, and three-layer fully connected back-propagation neural networks. Their results indicated that support vector machines outperformed other approaches Lee was trying to predict credit rating of a company using support vector machines. They used various financial indicator and ratios such as interest coverage ratio, ordinary income to total assets, Net income to stakeholders' equity, current liabilities ratio, etc. and achieved accuracy of around 60\%. Predicting credit rating of the companies were also studied using neural networks achieving accuracy between $75 \%$ and $80 \%$ for the United States and Taiwan markets Tsai and Wang

[2] Did a research where they tried to predict stock prices by using ensemble learning, composed of decision trees and artificial neural networks. They created dataset from Taiwanese stock market data, taking into account fundamental indexes, technical indexes, and macroeconomic indexes. The performance of Decision Tree + Artificial Neural Network trained on Taiwan stock exchange data showed F-score performance of 77\%. Single algorithms showed F-score performance up to $67 \%$.Kim and Han

[3] Guresen et al. (2011) describe ANN as one of the best techniques to model the stock market, because it does not contain standard formulas and may be easily adapted to market changes. ANN have the ability to learn by example and make interpolations and extrapolations of what they learned. The use of ANN in the solution of a task initially involves a learning phase, which is when the network extracts the patterns, thereby creating a specific representation of the problem (Braga, Carvalho, \& Ludermir, 2007).

The first model for prediction of stock price based on ANN was developed by White (1988). The author used a feed forward network to detect unknown regularities in stock price changes. The goal was to analyze the daily returns of IBM stock in order to test the efficient market theory, proposed by Fama (1970), which states that stock prices follow a random walk. Although he has not obtained good predictive results, the research stressed the potential for such analysis. Since then, a large number of researchers have actively participated in the development of predictive models that may be reliably applied in the stock market.

[4] Stock Market prediction has been one of the more active research areas in the past, given the obvious interest of a lot of major companies. In this research several machine learning techniques have been applied to varying degrees of success. However, stock forecasting is still severely limited due to its non-stationary, seasonal and in general unpredictable nature. Stock Market Prediction Using Hidden Markov Models Aditya Gupta, Non-Student Member, IEEE and Bhuwan Dhingra, Non-Student member, IEEE T Predicting forecasts from just the previous stock data is an even more challenging task since it ignores several outlying factors (such as the state of the company, economic conditions ownership etc.)

[5] A later study conducted by Tsibouris and Zeidenberg (1995) tests the efficient market theory by analyzing six stocks of the US stock exchange, finding a capacity to predict prices based on historical series of prices. Other studies, such as (Kolarik \& Rudorfer, 1994; Refenes, 1991; Refenes, Azema-Barac, \& Zapranis, 1993), observed that ANN obtain better prediction performance comparable to statistical techniques, such as the regression model and those obtained by ARIMA technique.

\section{PROPOSED SYSTEM}

The proposed model is based on the study of historical data, technical indicators and optimizing neural network algorithm to be used in the prediction of daily stock prices. The proposed model architecture contains six inputs vectors represent the historical data and derived technical indicators and one output represents next price. The following steps were used to build a neural model capable of making predictions of stocks closing prices short term future behaviour:

(1) understanding of the problem domain and identification of key variables;

(2) pre-selection and collection of samples;

(3) pre-processing of inputs;

(4) Modelling and prediction. 


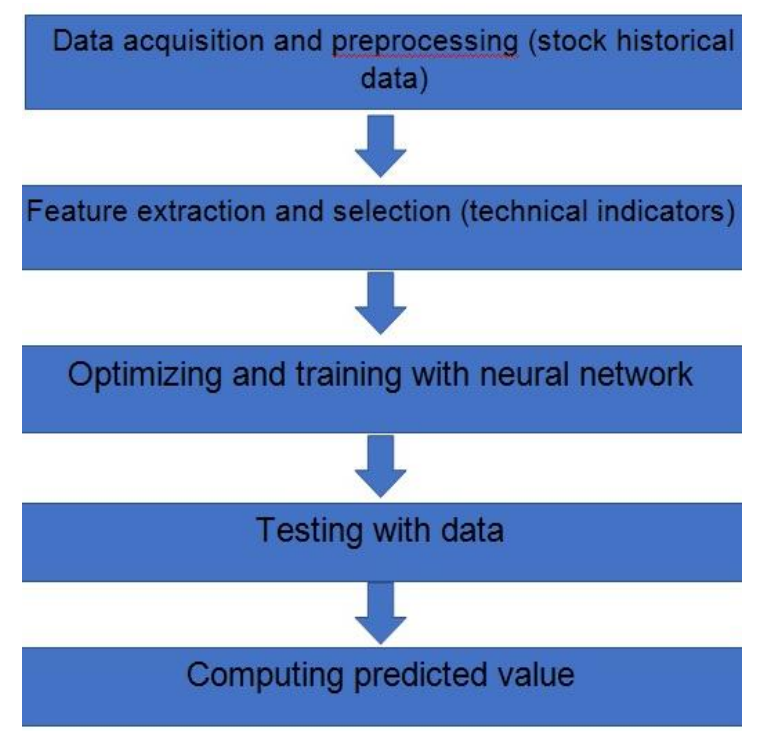

Fig. 1Proposed System

Fig. 1 shows the proposed architecture of the system. Artificial Neural Network (ANN) is an information processing system where the elements called neurons, process the information. The signals are transmitted by means of connection links. The links possess an associated weight, which is multiplied along with the incoming signal (net input) for any typical neural network. The output signal is obtained by applying activations to the net input. The network consists of a set of sensory units that constitute the input layer and one or more hidden layer of computation modes. The input signal passes through the network in the forward direction. This type of network is called as multilayer perception (MLP). The multilayer perception are used with supervised learning and have to lead the successful back propagation algorithm where logistic sigmoid function is widely used. The MLP network has hidden neurons and this will make the network more active for complex tasks. The layers of network are connected by synaptic weights and have a high computational efficiency.

\section{METHODOLOGY OF BUILDING A PREDICT MODEL}

General steps of building and predicting the value by using Multi Layer Perception model in the Neural Works Predict.

1. Building a Predict Model: To make predictions from data if target outputs can be any value in a

Continuous range of numeric values or a discrete ordered range of numeric values.

2. Selection of model: Multi Layer Perception (MLP) model is selected to predict the stock value.

3. MLP Input training data

4. MLP Output training data

5. MLP Training data characteristics

6. MLP Network parameters

7. Reviewing parameters and training the model

8. Saving the model

9. Training statistics

10. Testing a predict model

11. Specifying data sets for testing

12. Interpreting test results

13. Running a MLP predict model

\section{RESULTS}

Fig. 2 shows an example of an image with adequate resolution of Artificial Neural Network. A neural network is a model characterized by an activation function, which is used by interconnected information processing units to transform input into output. The first layer of the neural network of the neural network receives the raw input, process it and passes the processed information to the hidden layer. 


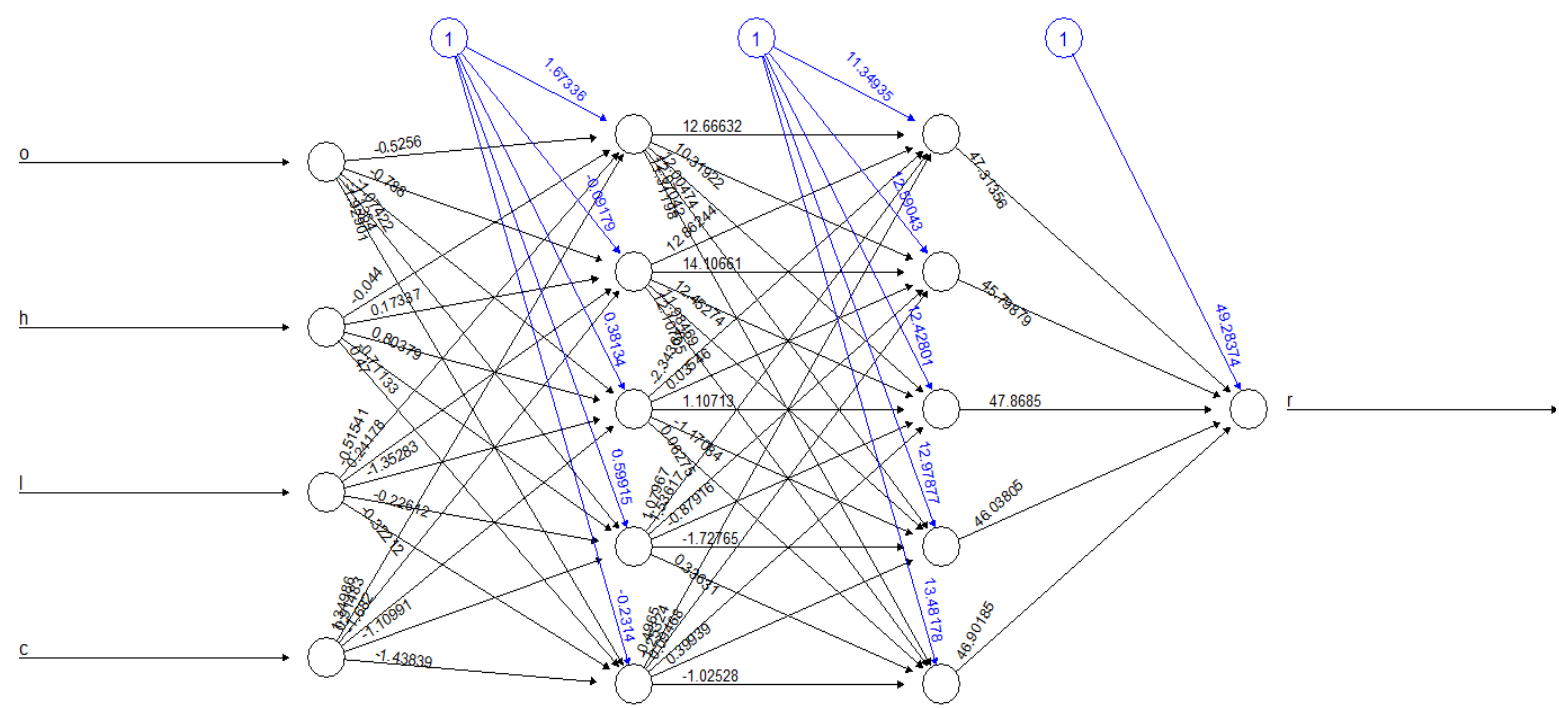

Fig. 2.Artificial Neural Network
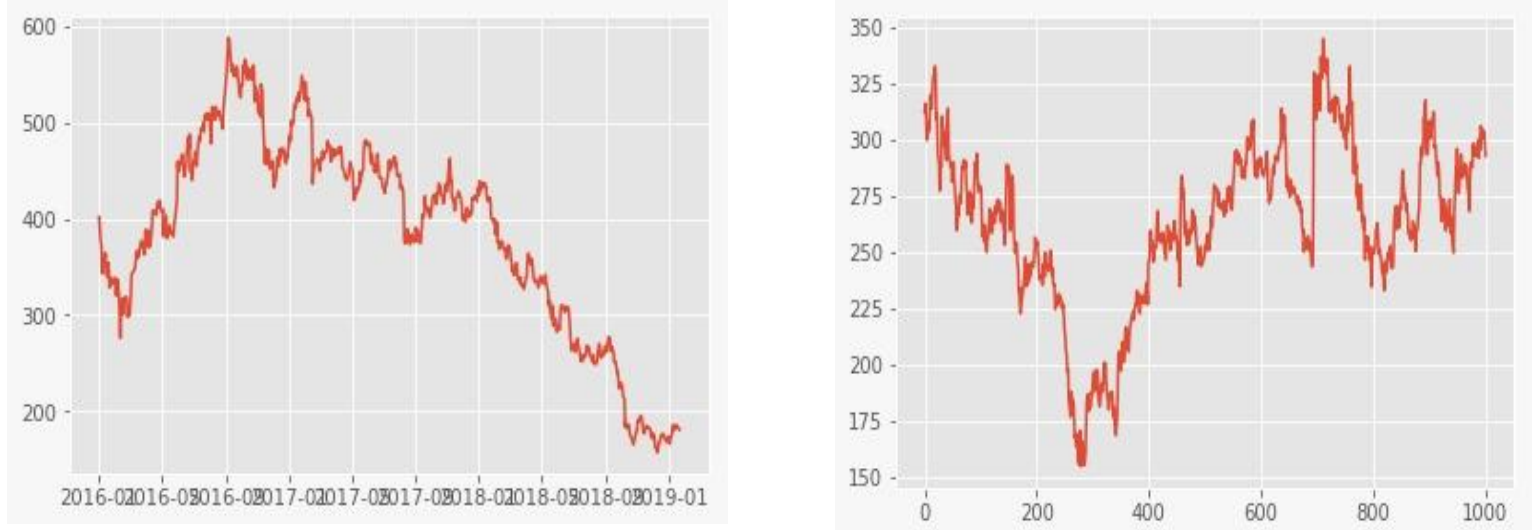

Fig 3(i)

Fig. 3(i) \& 3(ii).Stock Prediction Graph

Fig 3(ii)

In Fig 3(i) \&3(ii) shows the prediction graph using neural network. The data is of Tata Motors whereas in fig 3(i) shows the opening price from the Jan 2016 till Jan 2019. And the fig 3(ii) shows the closing price of Tata Motors. The data has been accessed from yahoo server.

\section{CONCLUSION}

In this research, we sought to understand the set of information present in the financial market and identify the variables that drive stock prices, taking into account the activities performed and economy sectors, the various industry, macroeconomic and market indicators. The methodology presented may be adapted to other enterprises and their stocks. Theoretically, the discussion on the predictions of stock prices is still controversial and unproductive. However, from the empirical standpoint, this research proved to be very productive, with some methods for financial market prediction being developed and demonstrated. Predicting the direction into which stock prices change is very important for the development of effective strategies to operate in the stock market. The result of the questionnaire applied to qualified respondents revealed that $80 \%$ of the respondents believe that the models and methods they use are satisfactory, but they do not use computer techniques to operate in the stock market. The assertive prediction of stock prices may promise attractive benefits for investors in general. In general, the results were satisfactory as they successfully achieved the objective of this research to predict the closing price. Thus, the use of ANN to predict the behavior and trends of stocks has shown to be a feasible alternative to conventional techniques, revealing the market behavior and offering privileged information to investors, in addition to the possibility of being used in combination with other techniques. 
Vol. 8, Issue 1, January 2019

\section{FUTURE SCOPE}

Neural networks often lead to significant results, e.g. in weather forecasting, a rule of weather change is less probable than a steady weather pattern. This is also true for stock prices. A key aspect to successful forecasting lies in the ability to merge data available in diverse formats. The data analysis performed by neural networks tolerates a considerable amount of imprecise and incomplete input data due to the distributed mode of information processing. Neural network lie in their ability to predict accurately even in situations with uncertain data, and the possible combinations with other methods. Despite the benefits of artificial neural networks, there are still some limitations to neural networks that are discussed below. Some methods are executed with insufficient reliability tests, data design and with inability to identify the optimal topology for a specific problem domain. There is no known method of designing an optimal neural network, but the best network is highly dependent on the data and application.

Some of the limitations are mentioned below:

1. NN require very large number of previous data.

2. The best NN architecture topology is still unknown.

3. For complex networks the result and accuracy may decrease.

4. Statistical relevance of the result is needed.

5. More careful data design is needed and systematically analyzed.

In order to improve the $\mathrm{NN}$ applications, there are some other limitations, concerning the problems of evaluation and implementation of NN that should be discussed. Large number of research is done and implemented by companies that are not published in scientific indexes.

\section{ACKNOWLEDGMENT}

We take an opportunity to acknowledge and extend our gratitude to our Project Guide Prof. Yogita Deshmukh Professor of Computer Technology, RGCER, Nagpur. She has helped us in many ways. Her enthusiastic engagements in our project work and her never-ending stream of ideas have been absolutely essential for the results, presented here. We are very grateful that she has spent so much time with us discussing different problems ranging from philosophical issues down to minute technical details. We would also like to express our sincere thanks to Prof. Yogita Deshmukh Asst. Professor of CT, Rajiv Gandhi College of Engineering and Research, Nagpur for her Guidance and kind support. We express our deepest sense of gratitude to Dr. Manali Kshirsagar, Principal, RGCER, Nagpur.

We also extend our thanks to all the faculty members of the department for providing their cooperation. Finally, we would like to thanks to everybody who has directly or indirectly contributed to our project work.

\section{REFERENCES}

[1]. K. Tsai and J. Wang, "External technology sourcing and innovation performance in LMT sectors", Research Policy, vol. 38, no. 3, pp. 518-526, 2009.

[2]. K. Han and J. Kim, "Genetic quantum algorithm and its application to combinatorial optimization problem", Evolutionary Computation, 2000, vol. 2, pp. 1354-1360, 2000.

[3]. Atsalakis, G. S., Dimitrakakis, E. M., \& Zopounidis, C. D. (2011). Elliott wave theory and neuro- fuzzy systems, in stock market prediction: The WASP system. Expert Systems with Applications, 38, 9196-9206

[4]. Olivier C., Blaise Pascal University: "Neural network modeling for stock movement prediction, state of art". 2007

[5]. Leng, X. and Miller, H.-G. : "Input dimension reduction for load forecasting based on support vector machines", IEEE International Conference on Electric Utility Deregulation, Restructuring and Power Technologies (DRPT2004), 2004.

[6]. Chun C, Qinghua M, Shuqiang L.: "Research on Support Vector Regression in the Stock Market Forecasting" CSpringer, Advances in Intelligent and Soft Computing Volume 148, , pp 607-612, 2012.

[7]. Guo Z., Wang H., Liu Q. : "Financial time series forecasting using LPP and SVM optimized by PSO" C Springer, Soft Computing Methodologies and Applications, December 2012.

[8]. Tsibouris, G., \& Zeidenberg, M. (1995). Testing the efficent markets hypothesis with gradient descent algorithms. In R A (Ed.), Neural networks in the capital markets.John Wiley and Sons.

[9]. Guresen, E., Kayakutlu, G., \& Daim, T. U. (2011). Using artificial neural network models in stock market index prediction. Expert Systems with Applications, 38(8), 10389-10397.

[10]. Gupta, A. :"Stock market prediction using Hidden Markov Models”, IEEE Engineering and Systems (SCES), 2012 Students Conference on, pp.1-4, 2012. 